\title{
Effect of friction on disoriented chiral condensate formation.
}

\author{
A. K. Chaudhurit \\ Variable Energy Cyclotron Centre \\ 1/AF,Bidhan Nagar, Calcutta - 700064
}

\begin{abstract}
We have investigated the effect of friction on the DCC domain formation. We solve the Newton equation of motion for the $\mathrm{O}(4)$ fields, with quenched initial condition. The initial fields are randomly distributed in a Gaussian form. In one dimensional expansion, on the average, large DCC domains can not be formed. However, in some particular orbits, large instabilities may occur. This possibility also greatly diminishes with the introduction of friction. But, if the friction is large, the system may be overdamped and then, there is a possibility of large DCC domain formation in some events.
\end{abstract}

25.75.+r, 12.38.Mh, 11.30.Rd

Typeset using REVTEX 
The possibility of forming disoriented chiral condensate (DCC) in relativistic heavy ion collisions has generated considerable research activities in recent years. The idea was first proposed by Rajagopal and Wilczek [1] 国]. They argued that for a second order chiral phase transition, the chiral condensate can become temporarily disoriented in the nonequilibrium conditions encountered in heavy ion collisions. As the temperature drops below $T_{c}$, the chiral symmetry begins to break by developing domains in which the chiral field is misaligned from its true vacuum value. The misaligned condensate has the same quark content and quantum numbers as do pions and essentially constitute a classical pion field. The system will finally relaxes to the true vacuum and in the process can emit coherent pions. Since the disoriented domains have well defined isospin orientation, the associated pions can exhibit novel centauro-like [5-8] fluctuations of neutral and charged pions [9 [12].

Most dynamical studies of DCC have been based on the linear sigma model, in which the chiral degrees of freedom are described by the real $\mathrm{O}(4)$ field $\Phi=(\sigma, \vec{\Pi})$, having the equation of motion,

$$
\left[\square+\lambda\left(\phi^{2}-v^{2}\right)\right] \Phi=H n_{\sigma}
$$

The parameters of the model can be fixed by specifying the pion decay constant, $f_{\pi}=92$ $\mathrm{MeV}$ and the meson masses, $m_{\pi}=135 \mathrm{MeV}$ and $m_{\sigma}=600 \mathrm{MeV}$, leading to $\lambda=\left(m_{\sigma}^{2}-\right.$ $\left.m_{\pi}^{2}\right) / 2 f_{\pi}^{2}=20.14$ and $v=\left[\left(m_{\sigma}^{2}-3 m_{\pi}^{2}\right) /\left(m_{\sigma}^{2}-m_{\pi}^{2}\right)\right]^{1 / 2} f_{\pi}=86.71 \mathrm{MeV}$ and $H=(120.55 \mathrm{MeV})^{3}$ [13]. It is apparent from eq.1] that the vacuum is aligned in the $\sigma$ direction $\Phi_{v a c}=\left(f_{\pi}, \mathbf{0}\right)$ and at low temperature the fluctuations represent nearly free $\sigma$ and $\pi$ mesons. At very high temperature well above $v$, the field fluctuations are centered near zero and approximate $\mathrm{O}(4)$ symmetry prevails.

It is instructive to decompose the chiral field,

$$
\Phi(r, t)=<\phi(t)>+\delta \phi(r, t)
$$

where $\langle\phi\rangle$ is the average over a suitable region of space and can be identified with the (local) order parameter and $\delta \phi$ are the semiclassical fluctuations and can be identified with quasi-particle excitations. Using eq.2 and taking the average of eq.17, the equation of motion for the mean fields in the Hartree approximation can be obtained as,

$$
\frac{\partial^{2}<\phi>}{\partial t^{2}}=\lambda\left(v^{2}-<\phi>^{2}-3<\delta \phi_{\|}^{2}>-<\delta \phi_{\perp}^{2}>\right)<\phi>+H n_{\sigma}
$$

where $\delta \phi_{\|}$is the component of the fluctuation parallel to $\langle\phi\rangle$ and $\delta \phi_{\perp}$ is the orthogonal component. This equation imply that the motion of the mean field is determined by the effective potential,

$$
V(<\phi>)=\frac{\lambda}{4}\left(<\phi>^{2}+3<\delta \phi_{\|}^{2}>+<\delta \phi_{\perp}^{2}>-v^{2}\right)
$$

which clearly differs from the zero temperature one in presence of fluctuations. By varying the fluctuations, chiral symmetry can be restored or spontaneously broken. It is also evident that the evolution of the order parameter critically depends on the initial values of the fluctuations. When $\delta^{2} \equiv\left(3<\delta \phi_{\|}^{2}>-<\delta \phi_{\perp}^{2}>\right) / 6$ is large enough the chiral symmetry is approximately (as $\mathrm{H} \neq 0$ ) restored. If the explicit chiral symmetry breaking term is neglected, 
the phase transition takes place at the critical fluctuations $\delta_{c}^{2} \equiv v^{2} / 6$. For $\delta^{2}<\delta_{c}^{2}$, the effective potential takes its minimum value at $\langle\phi\rangle=\left(\sigma_{e}, 0\right)$, where $\sigma_{e}$ depends on $\delta^{2}$. When the mean fields are displaced from this equilibrium point to the central lump of the Mexican hat $(<\phi>\sim 0)$, the effective mass square

$$
m^{2}=\lambda\left(v^{2}-<\phi>^{2}-3<\delta \phi_{\|}^{2}>-<\delta \phi_{\perp}^{2}>\right)
$$

will become negative and DCC can form. Since the domain size is directly related to the time scale, during which the effective mass remains negative, it strongly depends on the initial condition, $\delta^{2}$ and $\langle\phi\rangle$ of the system. In the one loop effective theory, the fluctuations can be replaced by $\left(T^{2} / 2\right)$, their counterpart in a finite temperature field theory [14,15].

Recently, Biro and Greiner [16], using the Langevin equation for the linear sigma model, investigated the interplay of friction and white noise on the evolution of the order parameter. In the $\phi^{4}$ theory, it can be shown explicitly that if the hard modes are integrated out on the two loop level, Langevin type of equation emerges for the propagation of long wavelength fields [17]. They found that in different realistic initial volumes ranging from 1 to 1000 $\mathrm{fm}^{3}$, the average evolution do not shows any sensible instability. However individual events sometime shows significant growth of fluctuations.

In the present paper, we will investigate the role of friction on the possible DCC domain formation. To this end, we solve eq.3, augmented by a Bjorken type of scaling expansion and Rayleigh dissipation term [19].

$$
\frac{\partial^{2}<\phi>}{\partial \tau^{2}}+\left(\frac{D}{\tau}+\eta\right) \frac{\partial<\phi>}{\partial \tau}=\lambda\left[v^{2}-<\phi>^{2}-T^{2} / 2\right]<\phi>+H n_{\sigma}
$$

In the weak coupling limit, the friction $\eta$ is related to the on-shell plasmon damping rate, $\eta \equiv 2 \gamma_{p l}$. In the standard $\phi^{4}$ theory, the plasmon damping rate can be calculated for the $\sigma$ and the $\Pi$ fields [17, 18]. Assuming that the meson masses are the same, the friction coefficient can be obtained as [16],

$$
\eta=2 \gamma_{p l}=\frac{9}{16 \pi^{3}} \lambda^{2} \frac{T^{2}}{m} f_{S p}\left(1-e^{-m / T}\right)
$$

where $f_{S p}=-\int_{1}^{x} d t \frac{\ln t}{t-1}$ is the Spence function. At $T=T_{c}=\sqrt{2 f_{\pi}^{2}-2 m_{\pi}^{2} / \lambda}=123 \mathrm{MeV}$, and if $m / T \simeq 1$, the friction $\eta=2.2 \mathrm{fm}^{-1}$. This friction may be too high. Therefore we also investigate scenarios with $1 / 2$ and $1 / 4$ of its value. We also neglect the temperature dependence of the friction coefficient.

We will solve the eq.6 with quenched initial condition, for one dimensional $(\mathrm{D}=1)$ expansion. As argued before, DCC formation depend critically on the initial condition. To reflect the uncertainty in the initial condition, the initial fields are randomly distributed to a Gaussian form with the following parameters,

$$
\begin{gathered}
<\phi>=0 \\
<\phi^{2}>-<\phi>^{2}=v^{2} / 6 \\
<\stackrel{\circ}{\phi}=0 \\
<\stackrel{\circ}{\phi}^{2}>-<\stackrel{\phi}{\phi}>^{2}=4 v^{2} / 6
\end{gathered}
$$


For each trajectories, we compute the characteristic quantity $m_{t}=\operatorname{sgn}\left(m^{2}\right) \sqrt{|m|}$. The phenomenon of long wavelength DCC amplification will occur whenever this characteristic quantity is negative. We also calculate the amplification coefficient defined as,

$$
G=\int|m| \Theta\left(-m^{2}\right) d t
$$

Amplification of unstable orbits are $\sim \exp (G)$.

In fig.1, we have shown the ensemble average of 1000 trajectories of the characteristic quantity $m_{t}$ (solid line). We have considered four different values for the friction coefficient, $\eta=0,0.5,1.1$ and $2.2 \mathrm{fm}^{-1}$. Also shown is the $m_{t}$ for the most unstable orbit i.e. the orbit for which the amplification coefficient $\mathrm{G}$ is maximum (the dashed line). For $\eta=0$, $<m_{t}>$ execute oscillation about its relaxed value of $140 \mathrm{MeV}$. It remain positive throughout the evolution. This indicate that in one dimensional expansion, on the average DCC like phenomena will not occur. The most unstable orbit shows nice oscillations with slowly diminishing amplitude, indicating possibility of large DCC domain formation in this particular event. Thus while on the average, one dimensional system do not exhibit DCC phenomena, in some particular event, large DCC formation can occur. These results are in agreement with the other calculations [20,16]. With the introduction of friction the results changes. For small friction $\eta=0.5 \mathrm{fm}^{-1},<m_{t}>$ do not oscillate much. It also remain positive throughout the evolution. Like the previous frictionless case, DCC phenomena is not expected on the average level. The most unstable orbit exhibit rapidly damping oscillation. It remains negative but for a short period of time. As size of DCC domain is directly related to the time during which $m_{t}$ remain negative, the result indicate that even for the most unstable orbit, large DCC domains can not be expected in this situation. Introduction of friction greatly diminishes the possibility of large DCC domain formation. With further increase of the friction result changes again. With $\eta=1.1 \mathrm{fm}^{-1},<m_{t}>$ do not show oscillation. But we find that it enters into the unstable region for a short duration of time. With moderately large friction, DCC phenomena can occur on average level also. The most unstable orbit shows a interesting behavior. Once $m_{t}$ reaches a negative value, it remains negative for a long duration, then relaxing to its stable value of $140 \mathrm{MeV}$. Similar behavior is seen for $\eta=2.2 \mathrm{fm}^{-1} . \quad m_{t}$ for the most unstable orbit stays negative for quite a long time $(\sim 15$ $\mathrm{fm}$ ), indicating possibility of a large DCC formation in this particular event. The results indicate that if the friction is sufficiently large, one can hope to form large DCC domains in some particular event. This result can be understood easily. With large $\eta$, the system is overdamped and once the system enters into unstable regime, it can not come out from it and remains unstable for long duration.

In order to check that the oscillations in $m_{t}$ are stable or not, we have performed the time series analysis according to Hurst [21,22] and obtained Hurst coefficient (H). Hurst coefficient varies between $0-1$. For a perfectly random data set it is 0.5 . If $H>0.5$, the data set is persistent and they are antipersistent if $H<.5$. For the persistent data set, if the trend or behaviour in the data set is increasing or decreasing over a certain unit interval of time, it will continue to increase or decrease over such an interval, but no such comment can be made about antipersistent orbits. The most unstable orbits shown in fig.1 are found to be persistent $(H>.5)$. In fig.2, we have shown the distribution of the Hurst coefficient for the 1000 trajectories calculated. For the frictionless system, quite a large number of 
trajectories are found to be antipersistent. The oscillation for these orbits may eventually die out. With increasing friction, this number decreases and with $\eta=2.2 \mathrm{fm}^{-1}$, we find all the trajectories are persistent.

The amplification coefficient $(\mathrm{G})$ is an important parameter in the theoretical analysis of DCC. Larger the G, larger is the DCC domain size. In fig.3, we have shown the distribution of $\mathrm{G}$ for all the 1000 trajectories as obtained in the present calculation. The distribution of the persistent trajectories $(H>.5)$, are also shown (the shaded region). It is evident that maximum unstability is obtained in the frictionless case, with $\mathrm{G}$ as large as 2.4. Frictionless case also shows marked difference when only the persistent trajectories are considered. For the persistent trajectories the distribution decreases continuously with $\mathrm{G}$, in contrast, when all the trajectories are considered, the number of trajectories remains nearly constant between $\mathrm{G}=.5-1.5$. With friction, as most of the trajectories are persistent, there is not much difference between the total distribution and the distribution of persistent trajectories. As is the case with frictionless case, we find that number of trajectories decreases continuously with increasing $\mathrm{G}$.

The pions from a DCC domain shows anomalous behavior, as they have definite isospin orientation. Defining the neutral to total pion ratio $f$

$$
f=\frac{\pi^{0}}{\pi^{+}+\pi^{-}+\pi^{0}}
$$

the probability to obtain a particular fraction $f$ in case of DCC formation can be obtained as [9, 11, 12,

$$
P(f)=\frac{1}{2 \sqrt{f}}
$$

incontrast to the binomial distribution peaked at $1 / 3$ for normal hadronic reaction. From eq.14, an equivalent probability that $f<f_{1}$ can be obtained,

$$
P\left(f<f_{1}\right)=\sqrt{f}
$$

Noting that the pion yield will be proportional to $\int \Pi^{2} \tau d \tau$, we have calculated this probability. The results are shown in fig.4 (solid line). The dashed lines are the probability distribution given by eq.15. While for frictionless and for small friction, the agreement between the two are reasonable, for moderate to high friction, they in very good agreement. It seems that the pions are all from DCC like events. This raises a question. Preceding analysis shows that it is not possible to obtain large DCC domain except in a few trajectories. Yet we obtain neutral to total pion ratio following the probability distribution of a DCC domain. This indicate that $1 / \sqrt{f}$ may not be good signal to identify the pions from DCC domains.

To conclude, we have investigated the effect of friction on the possible DCC domain formation. It was shown that while in one dimensional case, on average DCC formation is not possible, there is finite probability for DCC domain formation in a event by event basis. We also find that friction strongly inhibit large DCC formation even in a event by event basis. However, if the friction coefficient is sufficiently large, the system may become overdamped and then large DCC domain can be formed. 


\section{REFERENCES}

* e-mail address:akc@veccal.ernet.in

[1] K. Rajagopal and F. Wilczek, Nucl. Phys. B379, 395(1993).

[2] K. Rajagopal and F. Wilczek,Nucl. Phys. B404, 577(1993).

[3] K. Rajagopal,in Quark-Gluon Plasma 2, ed. R. Hwa, World Scientific (1995).

[4] F. Wilczek, hep-ph/9308341. Phys. Reports 65,151(1980).

[5] C. M. G. Lattes, Y. Fujimoto and S. Hasegawa, Phys. Reports, 154,247(1980).

[6] G. Arnison et al, Phys. Lett. B 122,189 (1983).

[7] G. J. Alner, Phys. Lett. B 180415,(1996); Phys. Reports 154,274 (1987).

[8] J. R. Ren et al, Phys. Rev. D 38,1517 (1988).

[9] A. A. Anselm and M. G. Ryskin , Phys. Lett. B 266,482 (1989).

[10] A. A. Aneslm, Phys. Lett. B217, 169(1989).

[11] J. D. Bjorken, K. L. Kowalski and C. C. Taylor, SLAC-PUB-6109 (1993), K.L. Kowalski K. L. and Taylor C. C., CWRUTH-92-6 (1992),hep-ph/9211282.

[12] J. P. Blaizot ,A. Krzywicki , Phys. Rev.D46, 246 (1992).

[13] J. Randrup, Report LBL-38125(1996), hep-ph/9602343.

[14] S. Gavin and B. Muller , Phys. Lett.B329,486 (1994).

[15] J. Randrup, Phys. Rev. D 55,1188(1997).

[16] T. S. Biro and C. Greiner, Phys. Rev. Lett. 79, 3138(1997).

[17] C. Greiner and B. Muller, Phys. Rev. D45, 1026 (1997).

[18] R. P. Parwani, Phys. Rev. D 45,4695(1992); S. Jeon, Phys. Rev. D.52,3591(1995).

[19] J. Randrup,hep-ph/9611228

[20] J. Randrup, Phys. Rev. Lett. 77,1226(1997).

[21] H. E. Hurst, Trans. Am. Soc. Civ. Eng.,116,770 (1951)

[22] H. E. Hurst, R. P. Black and Y. M. Simaika, Proc. IEEE, 1991, pp 3557. 


\section{FIGURES}

FIG. 1. Evolution of the ensemble averaged $m_{t}$ is shown for different friction coefficients (the

solid line). The most unstable orbit, for which the amplification coefficient is the largest is also shown (the dashed line).

FIG. 2. Histogram showing the distribution of Hurst coefficient for the 1000 trajectories.

FIG. 3. Distribution of the amplification factor G. The shaded distribution is for the persisten trajectories with Hurst coefficient $H>0.5$.

FIG. 4. The probability that the neutral to total pion ratio $f$ is less than $f_{1}$. The solid line is the calculated while the dashed line is from eq.15. 
FIGURES

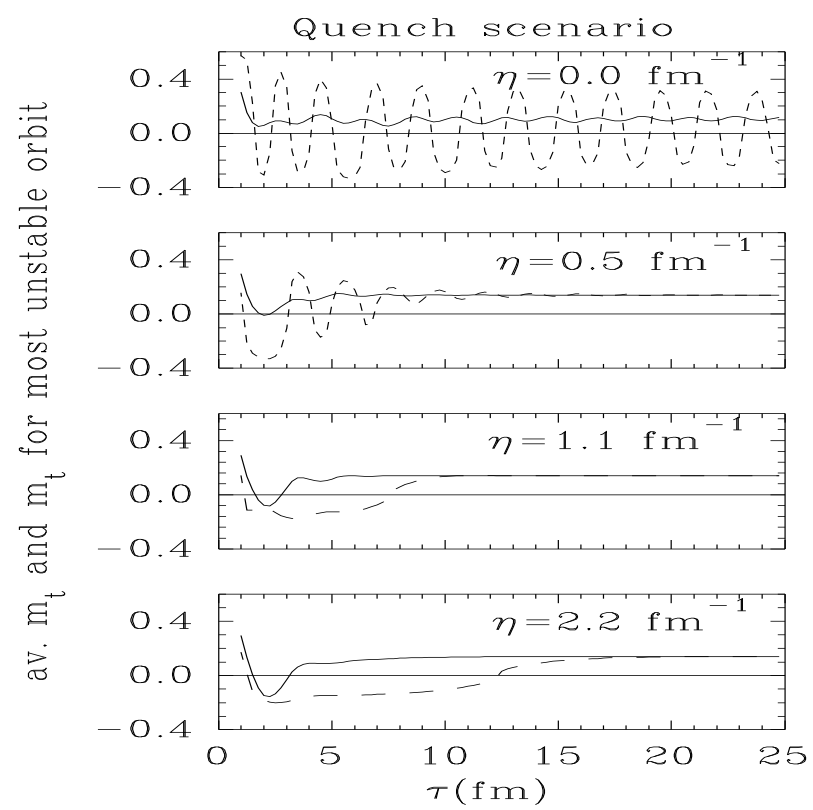

FIG. 1. Evolution of the ensemble averaged $m_{t}$ is shown for different friction coefficients (the solid line). The most unstable orbit, for which the amplification coefficient is the largest is also shown (the dashed line).

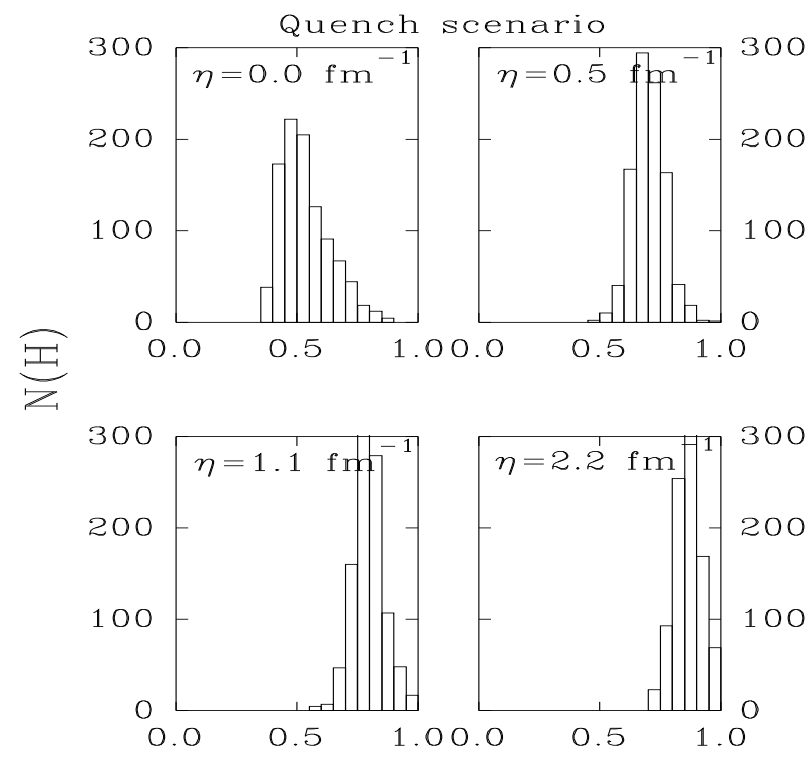

$\mathrm{H}$

FIG. 2. Histogram showing the distribution of Hurst coefficient for the 1000 trajectories. 


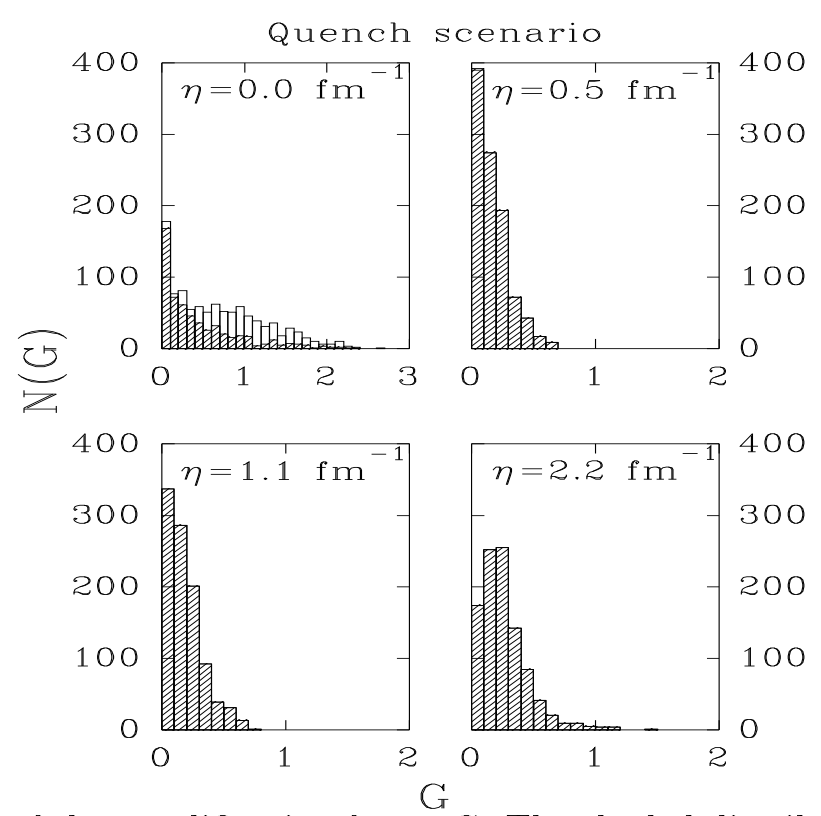

FIG. 3. Distribution of the amplification factor G. The shaded distribution is for the persisten trajectories with Hurst coefficient $H>0.5$.

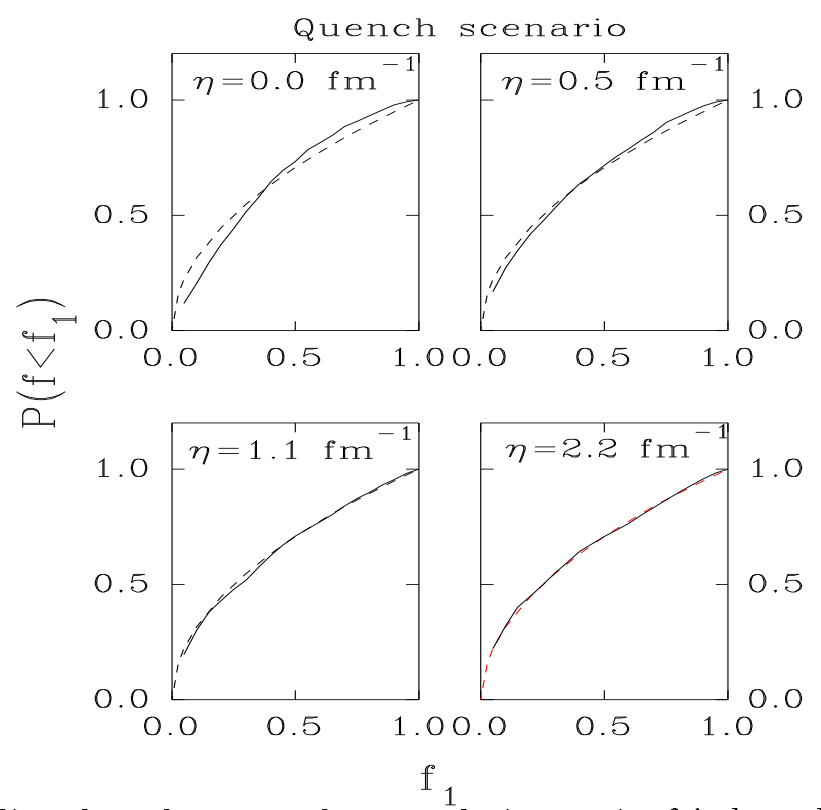

FIG. 4. The probability that the neutral to total pion ratio $f$ is less than $f_{1}$. The solid line is the calculated while the dashed line is for $\sqrt{f}$ probability 